\title{
Dimensiones de bienestar psicológico y apoyo social percibido con relación al sexo y nivel de estudio en universitarios
}

\author{
Dimensions of Psychological Well-Being and Perceived Social Support \\ in Relation to Sex and Level of Study in University Students \\ Dimensões de bem estar psicológico e apoio social percebido com relação \\ ao gênero e nível de estudo em universitários
}

\author{
Yarimar Rosa-Rodríguez, Nadjah Negrón Cartagena, Yazmín Maldonado Peña, \\ Areliz Quiñones Berrios, Neyra Toledo Osorio* \\ Universidad de Puerto Rico
}

Doi: dx.doi.org/10.12804/apl33.01.2015.03

\section{Resumen}

El modelo multidimensional de bienestar psicológico de Ryff ha permitido la evaluación del constructo desde seis dimensiones (autoaceptación, relaciones positivas con otros, autonomía, dominio del entorno, crecimiento personal y propósito en la vida). Existen pocas investigaciones entre el Bienestar Psicológico y el apoyo social, específicamente en poblaciones universitarias. Además, se encuentran pocos estudios sobre las diferencias por sexo y el nivel de estudio, con relación al Bienestar Psicológico y al apoyo social. Para evaluar la relación entre las dimensiones de bienestar psicológico y el apoyo social se utilizaron las Escalas de Bienestar Psicológico de Ryffy y el Cuestionario de Apoyo Social MOS. Se reclutó una muestra por disponibilidad de 768 estudiantes universitarios de pregrado y posgrado. Los resultados muestran correlaciones de bajas a moderadas, entre las Escalas de Bienestar Psicológico y las sub-escalas de Apoyo Social. Las mujeres exhiben promedios significativamente más altos en las escalas de crecimiento personal y propósito en la vida. El análisis de ANOVA simple y las respectivas pruebas de contraste indican que hay diferencias significativas entre los niveles de pregrado y doctorado para tres de las variables dependientes examinadas.

Palabras clave: Escalas de bienestar psicológico, apoyo social, estudiantes universitarios

* Yarimar Rosa-Rodríguez, Instituto de Investigación Psicológica, Universidad de Puerto Rico, Recinto de Río Piedras; Nadjah Negrón Cartagena, Departamento de Psicología, Universidad de Puerto Rico; Yazmín Maldonado Peña, Departamento de Psicología, Universidad de Puerto Rico; Neyra Toledo Osorio, Departamento de Psicología, Universidad de Puerto Rico; Areliz Quiñones Berrios, Departamento de Consejería y Orientación Estudiantil, Universidad de Puerto Rico, Recinto de Rio Piedras.

Las investigadoras extienden su agradecimiento a Raúl Camacho Johnson por su asistencia en el análisis de los datos.

La correspondencia relacionada con este artículo deber ser dirigida a Yarimar Rosa-Rodríguez. Correo electrónico: yarimar.rosa@ upr.edu

Para citar este artículo: Rosa-Rodríguez, Y., Negrón, C. N., Maldonado P. Y., Toledo O. N., \& Quiñones B. A. (2015). Dimensiones de bienestar psicológico y apoyo social percibido con relación al sexo y nivel de estudio en universitarios. Avances en Psicología Latinoamericana, 33(1), 31-43. doi: dx.doi.org/10.12804/ap133.01.2015.03 


\section{fibstract}

Ryff's multidimensional model of psychological well-being has allowed the evaluation of the construct from six dimensions (self-acceptance, positive relations with others, autonomy, environmental mastery, personal growth and purpose in life). There is little research on the relationship between psychological well-being and social support, specially with college students. In addition, there are few studies on sex differences and the level of education in relation to psychological well-being and social support. To assess the relationship between the level of psychological well-being and social support Ryff's Psychological Well-Being Scales and The MOS Social Support Questionnaire were used. We recruited a sample of 768 college undergraduates and graduates by availability. The results show low to moderate correlations between the Ryff Psychological Well-Being Scales and Social Support subscales. Women exhibited significantly higher means on sub-scales of personal growth and purpose in life. A one-way ANOVA analysis and its respective post-hoc test, indicate that there are significant differences between undergraduate and doctoral levels for three of the dependent variables examined.

Keywords: Psychological well-being scales, social support, university students

\section{Resumo}

O modelo multidimensional de bem estar psicológico de Ryff, tem permitido a avaliação do construto desde seis dimensões (auto aceitação, relações positivas com outros, autonomia, domínio do entorno, crescimento pessoal e propósito na vida). Existem poucas pesquisas entre o bem estar psicológico e o apoio social, especificamente em populações universitárias. Além disso, encontram-se poucos estudos sobre as diferenças por gênero e nível de estudo com relação ao bem estar psicológico e ao apoio social. Para avaliar a relação entre as dimensões de bem estar psicológico e o apoio social utilizamos as Escalas de Bem Estar Psicológico, bem estar psicológico de Ryff e o questionário de Apoio So- cial MOS. Recrutamos uma amostra por disponibilidade de 768 estudantes universitários de licenciatura e pósgraduação. Os resultados mostram correlações baixas ou moderadas entre as Escalas de Bem Estar Psicológico, bem estar psicológico e as subescalas de Apoio Social. As mulheres exibem meias significativamente maiores nas escalas de crescimento pessoal e propósito na vida. A análise de ANOVA simples e as respectivas provas de contraste indicam que tem diferenças significativas entre os níveis de licenciatura e doutoramento para três das variáveis dependentes examinadas.

Palavras-chave: Escalas de bem estar psicológico, apoio social, estudantes universitários

El estudio del bienestar se ha guiado básicamente por dos corrientes. La primera de ellas, el bienestar subjetivo, se refiere a la evaluación de los aspectos emocionales, afectivos y cognitivos, que a su vez incluyen el afecto positivo, el negativo y la satisfacción. Por otro lado, se encuentra la evaluación del funcionamiento óptimo de las personas, a la cual se le ha conocido como bienestar psicológico (Ryan \& Deci, 2001). Díaz, et al. (2006) definieron el Bienestar Psicológico como el desarrollo de las capacidades y el crecimiento personal, considerando estas últimas como los principales indicadores del funcionamiento positivo.

De estas dos corrientes, la del bienestar psicológico ha sido ampliamente estudiada, en especial desde la introducción del modelo multidimensional de Ryff (1989). El modelo propone seis dimensiones en un continuo positivo-negativo de evaluación de la percepción del individuo. La primera dimensión es la de auto-aceptación y se refiere a la evaluación positiva presente y pasada del individuo. La autonomía, como segunda dimensión, guarda relación con el sentido de autodeterminación y la independencia del sujeto. Por otro lado, la tercera dimensión, el crecimiento personal, está relacionada con el sentido de crecimiento y desarrollo como persona. La cuarta dimensión de Bienestar Psicológico, el propósito en la vida, se relaciona 
con la creencia de que la vida tiene algún significado y propósito para la persona. La dimensión de relaciones positivas con otros es la quinta y hace referencia a poseer o desarrollar relaciones de calidad con otros. Por último, la dimensión de dominio del entorno se refiere a la capacidad de manejar el ambiente donde se encuentra la persona y a utilizar efectivamente las oportunidades que este le brinda.

Este modelo ha sido estudiado por diversas áreas de la psicología y con variedad de poblaciones. En un repaso actualizado sobre el estado del modelo teórico, es la propia Carol Ryff quien identifica seis áreas esenciales de estudio en los pasados veinticinco años: (a) cambios en el curso de desarrollo humano, (b) correlatos con características de personalidad, (c) relación con experiencias de la vida cotidiana, (d) relación con el trabajo y actividades comunitarias, (e) relación con la salud y factores de riesgo e (f) intervención y aspectos clínicos (Ryff, 2013). Este trabajo de investigación se circunscribe a los relacionados con experiencias de la vida cotidiana y al curso del desarrollo humano, tomando como marco el trayecto por la vida universitaria.

\section{Bienestar Psicológico y estudiantes universitarios}

Múltiples investigaciones relacionadas con el bienestar psicológico han utilizado muestras compuestas por estudiantes universitarios. Ciertamente, conocer la percepción del Bienestar Psicológico en esta población permite que se puedan desarrollar intervenciones y programas de acuerdo con las necesidades específicas de los universitarios/as (Burris, Brechting, Salsman \& Carlson, 2009; Cooke, Bewick, Barkham, Bradley \& Audin, 2006). Se reconoce que las responsabilidades y deberes de un/a estudiante universitario son muy diferentes a las de otros miembros de la población en general, ya que estos están sometidos a constantes evaluaciones, tanto académicas como personales y sociales. Esto puede incidir en su rendimiento aca- démico, por lo que las universidades deben adoptar políticas educativas, estrategias y programas que permitan al estudiantado universitario responder de forma adecuada a estas situaciones (Velásquez et al., 2008).

Burris et al. (2009) indicaron que el momento de comenzar una carrera universitaria se distingue de otros periodos de desarrollo ya que este genera mucho estrés y ansiedad. Algunos problemas que pueden desarrollar los/as estudiantes en este periodo son ansiedad, trastornos alimentarios, fatiga y dificultad para concentrarse. Estos autores entendieron que el conocer los factores asociados con el bienestar psicológico en los/as estudiantes universitarios permitiría desarrollar estrategias de intervención que fuesen más efectivas, según las características particulares de cada estudiante. Por tal razón, decidieron identificar los factores individuales y conductuales que están asociados con la salud psicológica de los estudiantes universitarios. Entre los resultados más notables encontraron que el mejor predictor de Bienestar Psicológico en estudiantes universitarios es el optimismo, por lo que recomendaron que se inicien programas de prevención que le sean útiles al estudiante y que logren que este/a pueda afrontar los retos que se le presentan a diario en el contexto universitario. Además, indicaron que aquellos/as estudiantes que promueven un estilo de vida saludable, tienden a practicar conductas que pueden llevarlos a tener un mejor Bienestar Psicológico, así como a evitar conductas que pueden ser dañinas para su salud física, como por ejemplo consumir alcohol o utilizar drogas.

Por otra parte, Velásquez et al. (2008) entendieron que el Bienestar Psicológico junto con la asertividad y el rendimiento académico son variables que deben explorarse en la población universitaria con el fin de adoptar políticas educativas que brinden apoyo a estos/as. También, consideraron que es necesario tener más información respecto a la interacción de estas variables y la edad, sexo y facultad o carrera a la que pertenece el/la estudiante. 
Este grupo de investigadores halló una relación positiva entre bienestar psicológico y asertividad. Además, encontraron que el bienestar psicológico y el rendimiento académico se relacionan significativamente y que las mujeres puntuaron más alto en la escala de bienestar psicológico que los hombres.

Por otro lado, Bowman (2010) identificó cinco características que poseen los estudiantes, previo a su ingreso a la universidad como importantes contribuidores al desarrollo del bienestar psicológico: (a) ser de origen latino/a o hispano/a, (b) mujer, (c) entrar a una edad tradicional, (d) alto rendimiento académico y (e) tener aspiraciones previamente identificadas. En este estudio encontraron también que los estudiantes hispanos tenían niveles de bienestar psicológico superiores a los presentados por los estudiantes blancos no-hispanos de la muestra. Además, la edad parece ser un predictor importante para el desarrollo de Bienestar Psicológico una vez ingresan a la universidad. Por ejemplo, los estudiantes mayores de veinte años tienden a tener niveles más altos en la dimensión de propósito en la vida que los/as estudiantes que ingresan más jóvenes a la universidad.

Cooke et al. (2006) indicaron que las presiones sociales a las que están expuestos los estudiantes universitarios provocan peores condiciones de Bienestar Psicológico, si se compara con el resto de la población. Esto puede deberse, según los autores, a la presión que tiene el estudiante de obtener un grado académico y encontrar un trabajo que le permita solvencia económica una vez se gradúe. Los autores compararon el bienestar psicológico en tres grupos de jóvenes, justo antes de comenzar su primer año de universidad y en los primeros dos semestres académicos. Los resultados demostraron que el Bienestar Psicológico de este grupo de estudiantes no fue estático sino que cambió en las cuatro medidas obtenidas durante el año académico en que contestaron el cuestionario sobre Bienestar Psicológico. Esto sostiene la noción de que el Bienestar Psicológico es un continuo que cambia y varía según el desarrollo y los acontecimientos de la vida cotidiana. Otro hallazgo importante en dicha investigación es el hecho de que una cohorte de los estudiantes acudió con mayor frecuencia a los servicios de consejería ofrecidos por la universidad. En este sentido, una de las recomendaciones estriba en que las ayudas que se brinden en estos programas sean tanto formales como informales, de manera que aquellos que no acuden a las oficinas sí puedan tener acceso a cualquier otra información que les permita manejar los periodos de ansiedad durante su primer año de estudios universitarios $\mathrm{y}$, por ende, mantener niveles óptimos de Bienestar Psicológico.

Con relación a los cambios que pueden suscitarse en la percepción del bienestar en el curso de una etapa, Bewick, Koutsopoulou y Miles (2010) siguieron a un grupo de estudiantes durante varios años para evaluar el cambio suscitado en el Bienestar Psicológico. En su estudio encontraron que el Bienestar Psicológico de estudiantes universitarios reflejaba cambios cuando eran comparados con las puntuaciones de los estudiantes justo antes de entrar a la universidad.

Lee y Yuen Loke (2005) pretendieron identificar aquellas conductas saludables en estudiantes universitarios en Hong Kong, incluyendo variables como responsabilidad con su salud, actividad física y hábitos nutricionales. También, evaluaron el Bienestar Psicológico incluyendo variables como crecimiento espiritual, relaciones interpersonales y manejo del estrés. Utilizaron el Health Lifestyle Profile (HPLP-II) para medir las conductas saludables y el Bienestar Psicológico de 247 estudiantes. Uno de los hallazgos más importantes fue que hubo diferencias en cuanto al sexo del participante y las medidas de conductas saludables y Bienestar Psicológico. En este grupo, las mujeres tenían mayores puntuaciones en conductas saludables, mientras que los hombres hacían más actividad física. También, las mujeres fueron más capaces de utilizar sus relaciones interpersonales para mantener su Bienestar Psicológico, mientras que los hombres utilizaron más métodos para manejar el estrés que 
las mujeres. Por último, no encontraron diferencias entre el manejo del estrés y el crecimiento espiritual por sexo del participante.

Por último, García-Andalete (2013) analizó las diferencias en el Bienestar Psicológico, en función del sexo y la edad, de 180 estudiantes universitarios. Al utilizar la Escala de Bienestar Psicológico de Ryff encontró que las mujeres puntuaron más alto que los hombres en la dimensión de crecimiento personal, pero no en las dimensiones de relaciones positivas con otros, autonomía y propósito en la vida, como otras investigaciones habían señalado. El autor entendió que estos resultados están relacionados con cómo la sociedad esperaría que la mujer se preocupara por los demás y por trascender, mientras que los hombres están más enfocados en buscar nuevos objetivos, metas individuales y en la autopromoción.

Como se desprende de las investigaciones reseñadas, existe evidencia de que los niveles de Bienestar Psicológico varían de acuerdo con el sexo de un estudiante y el nivel de estudio en que se encuentre (comenzando estudios universitarios o más tarde en su preparación académica). En la medida en que puedan explorarse más a fondo estas diferencias, podrán generarse aplicaciones prácticas, tales como programas de mentoría, consejería, orientaciones y actividades de índole preventivo que permitan que los estudiantes universitarios tengan un mejor desempeño académico. Se considera que el sentido de Bienestar Psicológico puede ser clave para el éxito académico y el logro de las metas personales y profesionales de los estudiantes universitarios/as (Rosa-Rodríguez \& Quiñonez-Berrios, 2013).

\section{Apoyo social}

El Apoyo Social (AS) es un constructo amplio. La naturaleza multidimensional de este concepto ha requerido la clarificación de los diversos tipos de apoyo social y de los mecanismos que permiten que las necesidades afiliativas sean satisfechas (Kaplan, Cassel, \& Gore, 1977). En un intento de simplificar el concepto para ayudar a su comprensión, Lin, Woelfel y Light (1985) definieron el AS a partir de la Teoría de los Recursos Sociales, en términos de la fuerza de los lazos sociales y la tendencia de los individuos a asociarse y vincularse con otros similares. Por otro lado, Sherbourne y Stewart (Sherbourne et al., 1991) sugirieron desglosar el constructo en sus diversos componentes y evaluar cómo las diferentes dimensiones del AS se relacionan con efectos de la salud en el ser humano. En esta dirección, tales autoras sugieren dos vertientes principales del AS: apoyo estructural y apoyo funcional. El apoyo estructural se refiere a la existencia y cantidad de relaciones sociales y la interconexión de las redes sociales de una persona. El apoyo funcional, por otra parte, se refiere al grado en que las relaciones interpersonales sirven para funciones particulares. En esta vertiente se distinguen principalmente cinco tipos de apoyo: (a) emocional, (b) informacional, (c) tangible, (d) interacción social positiva y (e) apoyo afectivo. El apoyo emocional se refiere a las expresiones de afecto positivo, al entendimiento empático y a la estimulación de la expresión de sentimientos. El apoyo informacional aduce al asesoramiento y consejo, información, guía o retroalimentación que se ofrece a una persona. El apoyo tangible hace referencia a la provisión de ayuda material o asistencia recibida. La interacción social positiva se refiere a la disponibilidad de otros/as para hacer cosas divertidas y agradables con la persona. Finalmente, el apoyo afectivo involucra las expresiones de amor $\mathrm{y}$ afecto hacia una persona. Dichas investigadoras dan mayor énfasis a la percepción de un individuo sobre la ayuda con la que cuenta (o cree contar), por encima de la ayuda real recibida, destacando así la dimensión subjetiva del apoyo social.

Por otro lado, varios autores han hecho la distinción entre los aspectos objetivos del apoyo social (apoyo social recibido o real) y su dimensión subjetiva (apoyo social percibido). Según Barrón-López y Chacón-Fuertes (1992), ambas dimensiones del apoyo social (objetiva y subjetiva) se relacionan 
con el bienestar de los individuos. Kaplan, Casser y Gore (1977) destacan también la existencia de apoyo tangible (ayuda para la realización de una tarea) y apoyo evaluativo (ayudar en la redefinición de las expectativas del rol). Rodríguez-Espínola y Enrique (s. d.) elaboran sobre esta distinción al explicar que el Apoyo Social Objetivo (ASO) se refiere a los recursos, provisiones o transacciones reales disponibles para la persona al satisfacer sus necesidades, mientras que el apoyo social percibido (ASP) se refiere a la dimensión evaluativa en que la persona analiza la ayuda con la que cuenta o cree contar. Del mismo modo, Feldman et al. (2008) indican que el AS incluye elementos objetivos y subjetivos. Los primeros se relacionan con sucesos y los segundos con la percepción y con el significado que las personas le otorgan a estos.

Se entiende que el AS no se trata únicamente de la presencia de elementos tangibles recibidos por parte de otros/as, sino de la percepción misma que tenga la persona sobre estos. Tomando en consideración el carácter evaluativo de la percepción de los recursos accesibles, hallamos pertinente hacer una vinculación entre el ASP y el Bienestar Psicológico.

\section{Bienestar Psicológico y Apoyo Social Percibido}

Wang y Castañeda-Sound (2008) examinaron la influencia del ASP (de la familia y amigos) en el bienestar de estudiantes universitarios/as de primera generación, definiendo estos como aquellos/as universitarios/as cuyos padres no asistieron a una institución posgraduada. Según tales autores, el ASP es considerado como un factor importante del bienestar de los estudiantes universitarios/as de primera generación y las diversas fuentes de apoyo social (familia y amigos) que tienen implicaciones específicas sobre diversas áreas del bienestar (el estrés y la somatización). Adicional a ello, Feldman et al. (2008) exploraron la relación entre estrés académico, apoyo social, salud mental y rendimiento académico en una muestra de estudi- antes venezolanos/as. Los resultados de esta investigación indicaron que las condiciones favorables de salud mental están asociadas con un mayor AS y un menor estrés académico, reflejando diferencias por sexo con relación a la fuente de apoyo.

Por otro lado, Torres et al. (2010) han estudiado la relación del ASP y el autoconcepto en una muestra de 276 estudiantes de escuela superior. Los resultados reflejaron una correlación significativa entre ASP y autoconcepto, confirmando que a mayor ASP aumentan las puntuaciones de auto-concepto de los y las participantes. En estudios con poblaciones de mayor edad, Vivaldi y Barra (2012) examinaron la relevancia que tiene el ASP y la percepción de salud en el bienestar psicológico de adultos mayores. Estos demostraron que el ASP presenta relaciones significativas con tres dimensiones del Bienestar Psicológico (relaciones positivas, autoaceptación y propósito en la vida).

Ante la investigación que vincula al Bienestar Psicológico y el ASP se ha trazado este trabajo con el objetivo de evaluar la relación entre bienestar psicológico según las Escalas de Bienestar Psicológico y las sub-escalas de ASP del MOS. Como segundo objetivo, se examinaron las diferencias en la percepción de bienestar psicológico y ASP según el sexo y el nivel de estudio.

\section{Método}

Para el presente estudio se utilizó una metodología cuantitativa inferencial de tipo transversal.

\section{Participantes}

La muestra estuvo compuesta por 768 estudiantes universitarios de 17 a 74 años de edad $(M=21.74 ; S D=5.17)$. La muestra incluyó estudiantes de pregrado $(80.1 \%)$ y posgrado $(19.1 \%)$, provenientes de instituciones públicas $(88.3 \%)$ y privadas $(11.7 \%)$. Además, el $36.6 \%$ de 10 s participantes eran hombres $(n=281)$ y el $63 \%$ mujeres $(n=484)$. El $80 \%(n=615)$ de los y las 
participantes se encontraban cursando el pregrado al momento del estudio mientras el restante $20 \%$ $(n=147)$ se distribuye entre la/los niveles de escuela profesional, ${ }^{1}$ maestría y doctorado que pertenecen al posgrado. Seis participantes no respondieron a la pregunta sobre el nivel académico.

\section{Procedimiento}

Luego de obtener la autorización del Comité de Protección de Sujetos Humanos en la Investigación de la Universidad de Puerto Rico, Recinto de Río Piedras, se reclutó a los y las participantes en los salones de clase de distintas facultades académicas y escuelas. Profesores y profesoras en las distintas instituciones autorizaron el uso del espacio de curso para estos fines. Las y los profesores que autorizaron nuestra participación en sus secciones de clase proveyeron un espacio de treinta minutos aproximadamente, inhibiéndose de entrar al aula. Las asistentes de investigación, debidamente adiestradas, discutieron la hoja de consentimiento y distribuyeron los cuestionarios que se respondían en forma anónima, sin ningún tipo de identificadores directos, a aquellos estudiantes que accedieron a participar. Los y las estudiantes que declinaron su participación se retiraron del salón.

\section{Instrumentos}

Hoja de Datos Sociodemográficos. Esta sección recoge información que permite describir la muestra en términos de la edad, sexo, ingresos económicos, nivel y área de estudios.

\section{Escalas de Bienestar Psicológico de Ryff (EBP)} (Ryff, 1989). Se utilizaron las Escalas de Bienestar Psicológico (Ryff, 1989) en español, traducida por Díaz et al. (2006) en su versión de 29 reactivos, validada por van Dierendonck et al. (2007). La escala fue revisada por dos expertas en el uso del idioma español para asegurar su correspondencia lingüística con el lenguaje en Puerto Rico. De esta revisión solo se recomendó cambiar el voceo, que no es utilizado en Puerto Rico.

Las escalas se distribuyen en seis dimensiones y utilizan una escala Likert de seis puntos que va desde totalmente en desacuerdo hasta totalmente de acuerdo. Esta permite el cálculo de las puntuaciones para cada dimensión.

\section{Cuestionario MOS de Apoyo Social Percibido.}

Se utilizó una versión en español del cuestionario MOS de Apoyo Social Percibido (ASP), realizada por Rodríguez-Espínola y Enrique (2007). Esta versión fue evaluada por expertas de la misma manera que la EBP. La versión original del Cuestionario MOS fue desarrollada por Sherbourne y Stewart (1991) y distingue cinco tipos de apoyo social: emocional, informacional, instrumental, interacción social positiva y afectivo. Según las autoras, los resultados del análisis de factores reflejaron un traslapo considerable entre los reactivos de la dimensión emocional e informacional. Por tanto, la versión original del MOS distingue cuatro dimensiones principales: (a) emocional/informacional (AE), (b) instrumental (AI), (c) interacción social positiva (ISP) y (d) apoyo afectivo (AA).

El MOS contiene veinte reactivos. El primer reactivo hace referencia al apoyo estructural, es decir, al tamaño de la red social (cantidad de amigos/as o familiares cercanos con que cuenta la persona). Los reactivos dos al veinte se refieren a las cuatro dimensiones de apoyo social funcional antes mencionadas. Mediante una escala Likert de cinco puntos, el MOS explora con qué frecuencia la persona dispone del tipo de ayuda que se le presenta en la premisa. Puede obtenerse la puntuación total de la escala, de manera que, a mayor

1 El nivel de escuela profesional comprende aquellas disciplinas que se encuentran fuera de facultades académicas. En este caso incluye Escuela de Derecho y Escuela de Arquitectura. 
puntuación, mayor será el ASP. También pueden computarse puntuaciones totales para cada una de las sub-escalas.

\section{Análisis}

Se analizaron los datos utilizando SPSS 20 versión Windows. Específicamente, se estimó la consistencia interna de ambas escalas mediante el alfa de Cronbach. Se procedió con las estadísticas descriptivas y los análisis inferenciales incluyendo prueba $t$ para muestras independientes entre ESP, ASP y el sexo de los participantes. Se finalizó con ANOVA simple para ESP, ASP y los cuatro niveles de estudio así como las respectivas pruebas de contraste para determinar cuáles grupos difieren significativamente.

\section{Resultados}

\section{Consistencia interna de las escalas}

El análisis de consistencia interna de las EBP en este estudio está presentado en la tabla 1 . Como se indica, los valores alfa van de $.47 \mathrm{a} .80$ en las EBP, siendo las más bajas dominio del entorno $(\alpha=.47)$ y crecimiento personal $(\alpha=.59)$. Los valores más altos se encuentran en las escalas de autoaceptación $(\alpha=.74)$, relaciones positivas con otros $(\alpha=.71)$, autonomía $(\alpha=.60)$, y propósito en la vida $(\alpha=.80)$. Estos resultados son similares a otros estudios realizados con la escala de 29 y 39 reactivos (véase Díaz et al., 2006; Garcia-Alandete, Lozano, Nohales, \& Martínez, 2013; García-Andalete, 2013; Rodríguez-Carvajal, Diaz Méndez, Moreno-Jiménez, Blanco Abarca, \& van Dierendonck, 2010). El MOS obtuvo una consistencia interna de .84 (véase tabla 1). La consistencia de la escala total se acerca a la de las validaciones de Argentina (Rodriguez-Espinola \& Enrique, s. d.) y Colombia (Londoño Arredondo et al., 2012). Los valores alfa para las sub-escalas van de .171 (AA) a .941 (AE). Debido a la baja consistencia de la sub-escala de apoyo afectivo, esta no fue considerada para los análisis.

Tabla 1

Confiabilidad, promedios y desviaciones medias de Escalas de Bienestar Psicológico y MOS

\begin{tabular}{lrrr}
\hline \multicolumn{1}{c}{ Dimensión } & $\mathrm{A}$ & $M$ & $S D$ \\
\hline Escalas de Bienestar Psicológico & & & \\
Autoaceptación & .749 & 20.6 & 3.32 \\
Relaciones Positivas con otros & .715 & 23.34 & 4.85 \\
Autonomía & .603 & 26.93 & 4.84 \\
Dominio del entorno & .476 & 22.97 & 3.99 \\
Crecimiento personal & .594 & 21.23 & 2.77 \\
Propósito en la vida & .803 & 25.16 & 18.18 \\
MOS & & & \\
Total & .846 & 80.04 & 15.31 \\
Apoyo emocional & .941 & 33.05 & 7.05 \\
Apoyo instrumental & .449 & 16.29 & 5.23 \\
Interacción social positiva & .874 & 17.18 & 3.12 \\
\hline
\end{tabular}

\section{Correlaciones entre las escalas}

En la tabla 2 se presentan las correlaciones Spearman rho para las dimensiones de EBP y el MOS. Las correlaciones van de bajas a moderadas, todas con un nivel de significancia de $\underline{p} .<.0001$. La escala de autoaceptación obtiene correlaciones positivas y moderadas con AE ( $\rho=.364)$, e ISP $(\rho=.369)$. Relaciones positivas con otros obtienen correlaciones moderas con apoyo emocional $(\rho=.525)$, apoyo instrumental $(\rho=.302)$, e interacción social positiva $(\rho=.466)$. Por último, la escala de propósito en la vida obtiene correlaciones moderadas con $\operatorname{AE~}(\rho=.305)$ e ISP $(\rho=.321)$.

\section{Diferencias por sexo}

Se realizaron pruebas $t$ para examinar las diferencias entre hombres y mujeres. Las mujeres exhiben niveles más altos de autoaceptación 
Tabla 2

Correlaciones Spearmanrho para las Escalas de Bienestar Psicológico y Apoyo Social

\begin{tabular}{|c|c|c|c|}
\hline Dimensión & $\begin{array}{c}\text { Apoyo } \\
\text { Emocio- } \\
\text { nal }\end{array}$ & $\begin{array}{l}\text { Apoyo } \\
\text { instru- } \\
\text { mental }\end{array}$ & $\begin{array}{l}\text { Interac- } \\
\text { ción social } \\
\text { positiva }\end{array}$ \\
\hline Autoaceptación & $.364 *$ & $.253 *$ & $.369 *$ \\
\hline $\begin{array}{l}\text { Relaciones positivas } \\
\text { con otros }\end{array}$ & $.525^{*}$ & $.302 *$ & $.466^{*}$ \\
\hline Autonomía & $.260 *$ & $.211 *$ & $.258^{*}$ \\
\hline Dominio del entorno & $.278^{*}$ & $.205 *$ & $.259 *$ \\
\hline Crecimiento personal & $.245^{*}$ & $.187 *$ & $.210^{*}$ \\
\hline Propósito en la vida & $.305^{*}$ & $.230 *$ & $.321 *$ \\
\hline
\end{tabular}

$(M=19.96, S D=3.50)$, dominio del entorno $(M=22.92, S D=4.12)$, apoyo emocional $(M=$ 33.02, $S D=6.92)$, apoyo instrumental $(M=16.23$, $S D=3.97)$ y apoyo afectivo $(M=13.37, S D=5.11)$. Encontramos que las mujeres exhiben niveles significativamente más altos en crecimiento personal $(M=21.27, S D=4.12), t(762)=-1.93 . \underline{p} .<.05) . \mathrm{La}$ magnitud de la diferencia de los promedios $(M D$
$=-.442,95 \%$ CI: -.891--.007) resultó ser pequeña (Cohen's $d=.14$ ). Igualmente, las mujeres reportan niveles significativamente más altos en propósito en la vida $t(762)=2.24, p .<.02)$. La magnitud de la diferencia de los promedios $(M D=-.749,95 \% C I$ : -1.404- -.095) resultó ser pequeña (Cohen's $d=.2$ )

Mientras, los hombres exhiben niveles más altos en relaciones positivas con otros $(M=23.61$, $S D=4.60)$, autonomía $(M=27.14, S D=4.60)$, e interacción social positiva $(M=17.11, S D=3.32)$. No obstante, ninguna de estas diferencias resultó estadísticamente significativa. Para las sub-escalas de AS no se encontraron diferencias significativas.

\section{Diferencias por nivel de estudio}

Se realizó un análisis de ANOVA simple para explorar las diferencias por nivel de estudio en las dimensiones de EBP y AS. Los y las participantes fueron clasificados en cuatro niveles de estudios, un nivel para el pregrado y tres para posgrado (maestría, doctorado y escuela profesional). Encontramos diferencias significativas para autoaceptación $[F(3,757)=5.71, p<.001]$; relaciones

Tabla 3

Prueba t para sexo del participante, Escalas de Bienestar Psicológico y Apoyo Social

\begin{tabular}{|c|c|c|c|c|c|c|}
\hline \multirow[b]{2}{*}{ Dimensiones } & \multicolumn{2}{|c|}{ Hombres } & \multicolumn{2}{|c|}{ Mujeres } & \multirow[t]{2}{*}{$t(g l)$} & \multirow[t]{2}{*}{ Cohen's d } \\
\hline & $M$ & $S D$ & $M$ & $S D$ & & \\
\hline Escala de Bienestar Psicológico & & & & & $(762)$ & \\
\hline Autoaceptación & 19.95 & 3.30 & 19.96 & 3.50 & -.064 & \\
\hline Relaciones Positivas con otros & 23.61 & 4.60 & 23.07 & 5.04 & 1.46 & \\
\hline Autonomía & 27.14 & 4.60 & 26.58 & 5.04 & 1.52 & \\
\hline Dominio del entorno & 22.41 & 4.14 & 22.92 & 4.12 & -1.62 & \\
\hline Crecimiento personal & 20.83 & 2.99 & 21.27 & 3.07 & $-1.93 *$ & .1 \\
\hline Propósito en la vida & 24.50 & 4.52 & 25.25 & 4.39 & $-2.24 * *$ & .2 \\
\hline MOS & & & & & $(757)$ & \\
\hline Apoyo emocional & 32.71 & 7.32 & 33.02 & 6.92 & -5.75 & \\
\hline Apoyo instrumental & 16.18 & 6.89 & 16.23 & 3.97 & -.138 & \\
\hline Interacción social positiva & 17.11 & 3.32 & 17.00 & 3.19 & .433 & \\
\hline
\end{tabular}

$* p .<.05 ; * * p .<.01$ 
positivas con otros $[F(3,757)=4.39, p .<004]$; dominio del entorno $[F(3,757)=2.99, p .<.03]$; propósito en la vida $[F(3,757)=3.63, p .<.01]$, e interacción social positiva $[F(3,752)=3.31, p .<$ .02]. Las comparaciones post-hoc de Bonferroni y Games-Howell indican que los promedios del nivel de pregrado y doctorado difieren significativamente $(\underline{p} .<.05)$ en tres de las dimensiones evaluadas: relaciones positivas con otros, dominio del entorno e interacción social positiva. Siendo los estudiantes doctorales los que tienen promedios más altos en estas tres dimensiones.

\section{Discusión}

Este trabajo tenía como objetivo evaluar la relación entre las EBP y el ASP en una muestra de estudiantes universitarios. Además, evaluar las diferencias por sexo y nivel de estudio.

Se optó por utilizar las EBP en su versión española validada y reducida a 29 reactivos por Díaz et al. (2006) y van Dierendonck (2007). El análisis de consistencia de esta escala mostró niveles que van de bajos a altos. Ambas escalas muestran niveles de confiabilidad aceptables y comparables con otros estudios. Aunque los valores obtenidos son similares a los de otros trabajos, que utilizan la misma versión de la escala, las bajas puntuaciones en las dimensiones de dominio del entorno y crecimiento personal apuntan a la necesidad de evaluar la estructura factorial de esta. El MOS exhibe un índice de consistencia interna adecuado para su uso en tres de sus cuatro sub-escalas. La sub-escala de apoyo afectivo requiere una evaluación adicional a sus reactivos debido a la baja puntuación.

El análisis de correlación confirma la relación que existe entre todas las dimensiones de la EBP y AS. En especial la dimensión de relaciones positivas con otros con AE e ISP. El AE se refiere al afecto, la empatía y los sentimientos, por lo que se entiende que existe una vinculación entre la percepción del Bienestar Psicológico y los recursos que la persona entiende que tiene para llenar esta necesidad por medio de las interacciones y las relaciones con sus pares. En el contexto universitario, esto es de vital importancia, toda vez que al ingreso los y las estudiantes se ven obligados a desarrollar nuevas redes sociales que le acompañen durante esta etapa de su ciclo vital.

En términos de las diferencias por sexo, se observó que las mujeres obtienen promedios más altos en siete de las nueve dimensiones incluidas

Tabla 4

Promedios, desviaciones y ANOVA para Escalas de Bienestar Psicológico, Apoyo Social y Cuatro Niveles de Estudio

\begin{tabular}{lccccccccccc}
\hline \multicolumn{1}{c}{ Sub-escalas } & \multicolumn{2}{c}{ Pregrado } & \multicolumn{2}{c}{ Maestría } & \multicolumn{2}{c}{ Doctorado } & \multicolumn{2}{c}{ Escuela Profesional } & \multicolumn{2}{c}{ Anova } \\
\cline { 2 - 10 } & $M$ & $S D$ & $M$ & $S D$ & $M$ & $S D$ & $M$ & $S D$ & $F$ & Sig. \\
\hline Autoaceptación & 19.95 & 3.357 & 19.59 & 3.978 & 20.62 & 3.046 & 16.27 & 5.387 & 5.718 & .001 \\
Relaciones Positivas & 23.00 & 5.000 & 24.08 & 4.636 & 24.76 & 3.886 & 21.73 & 6.018 & 4.399 & .004 \\
Autonomía & 26.94 & 4.880 & 26.18 & 4.989 & 26.60 & 4.607 & 23.36 & 6.376 & 2.264 & .080 \\
Dominio del entorno & 22.59 & 4.066 & 22.56 & 4.627 & 23.86 & 4.126 & 21.55 & 4.503 & 2.990 & .030 \\
Crecimiento Personal & 21.10 & 3.002 & 21.36 & 3.376 & 21.34 & 2.665 & 20.09 & 5.394 & .678 & .566 \\
Propósito en la Vida & 24.95 & 4.377 & 23.90 & 5.051 & 25.95 & 4.241 & 22.36 & 5.801 & 3.630 & .013 \\
Apoyo Emocional & 32.65 & 7.145 & 32.97 & 7.103 & 34.36 & 6.515 & 32.40 & 6.703 & 1.654 & .175 \\
Apoyo Instrumental & 16.10 & 5.495 & 15.79 & 4.143 & 16.79 & 3.976 & 16.80 & 4.367 & .602 & .614 \\
Apoyo Interacción & 16.89 & 3.313 & 17.21 & 2.957 & 17.96 & 2.630 & 16.30 & 3.592 & 3.311 & .020 \\
Social Positiva & & & & & & & & & &
\end{tabular}


(seis de EBP y tres de ASP). Estos resultados se asemejan parcialmente a los de otros estudios, si se toman por ejemplo las puntuaciones en la escala de propósito en la vida y el sexo de los y las participantes. Por un lado, Ryff, Keyes y Hughes (2003) encontraron que las mujeres obtienen puntuaciones significativamente más altas en propósito en la vida y autonomía. Mientras, Lindfords, Berntsson y Lundberg (2006) encontraron que las mujeres exhibían promedios significativamente más altos en propósito en la vida y relaciones positivas con otros. En este estudio se exhiben promedios significativamente más altos que los hombres en crecimiento personal y propósito en la vida. Esto destaca la consistencia de este patrón en muestras diversas y con extracciones culturales distintas. Las diferencias significativas en la prueba $t$, también pueden ser explicadas por las expectativas asignadas a hombres y mujeres en cuanto a los roles de género. Los altos promedios en las mujeres en las dimensiones de autoconcepto, dominio del entorno, crecimiento personal y propósito en la vida y las sub-escalas de apoyo emocional, apoyo instrumental y apoyo afectivo son indicadores de una inclinación al cuidado de otros a la búsqueda de alcanzar metas más altas. Por otro lado, se observó que los promedios más altos de los hombres son en aquellas dimensiones y sub-escalas que se refieren a la búsqueda de recursos externos, en contacto con otras personas y al sentido de independencia, valores altamente apreciados culturalmente en los varones. En este sentido, este trabajo no examina la adherencia a los roles de género por lo que el alcance de las conclusiones es limitado. No obstante, esta información permite abrir una línea de investigación en donde se puede examinar la carga de los roles de género en la percepción del Bienestar Psicológico.

Por último, cuando se observan las diferencias según el nivel de estudios, se ve cómo el AS se hace más necesario a medida que los y las estudiantes avanzan a niveles más avanzados en su carrera, en especial el apoyo relacionado a la cercanía de los pares. No obstante, se hace necesario examinar las medidas de apoyo afectivo, ya que la escala utilizada no provee niveles de consistencia apropiados con esta muestra. Se puede destacar también como una limitación el tamaño de la muestra para los subgrupos de maestría, doctorado y escuela profesional. Las diferencias identificadas en este estudio son un indicador de la brecha que puede haber entre la transición del nivel de bachillerato al posgrado y que debe ser atendida desde una perspectiva de desarrollo.

\section{Referencias}

Barrón L. de R. A., \& Chacón F. F. (1992). Apoyo social percibido: Su efecto protector frente a los acontecimientos vitales estresantes. Aprendizaje, Revista de Psicología Social, 7(1), 53-59.

Bewick, B., Koutsopoulou, G., \& Miles, J. (2010). Changes in undergraduate students' psychological well-being as they progress through university. Studies in Higher Education, 35(6), 633-645.

Bowman, N. A. (2010). The development of psychological well-being among first-year college students. Journal of College Student Development, 51(2), 180-200. doi:10.1353/csd.0.0118

Burris, J. L., Brechting, E. H., Salsman, J., \& Carlson, C. R. (2009). Factors associated with the psychological well-being and distress of university students. Journal of American College Health, 57(5), 536-544.

Cooke, R., Bewick, B., Barkham, M., Bradley, M., \& Audin, K. (2006). Measuring, monitoring and managing the psychological well-being of first year university students. British Journal of Guidance and Counselling, 34(4), 505-517. doi:10.1080/03069880600942624

Díaz, D., Rodríguez-Carvajal, R., Blanco, A., Moreno-Jiménez, B., Gallardo, I., Valle, C., \& van Dierendonck, D. (2006). Adaptación española de las Escalas de Bienestar Psicológico Ryff. Psicothema, 18(3), 572-577. Recuperado 
de http://www.psicothema.com/psicothema. asp?id $=3255$

Feldman, L., Gonçalves, L., Puignau, G. C. C., Zaragoza, J., Bagés, N., \& De Paulo, J. (2008). Relaciones entre estrés académico, apoyo social, salud mental y rendimiento académico en estudiantes universitarios venezolanos. Universitas Psychologica, 7(3), 739-752.

García-Alandete, J., Lozano, B. S., Nohales, P. S., \& Martinez, E. R. (2013). Predictive role of meaning in life on psychological well-being and gender specific diferences. Acta Colombiana de Psicología, 16(1), 17-24.

García-Andalete, J. (2013). Bienestar psicológico, edad y género en universitarios españoles. Salud \& Sociedad, 4(1), 48-58. Recuperado de http:// www.saludysociedad.cl/index.php/main/article/ viewArticle/118

Kaplan, B., Cassel, J. C., \& Gore, S. (1977). Social support and health. Medical Care, 15(5), 47-58.

Lee, R. L. T., \& Yuen Loke, A. (2005). Health-promoting behaviors and psychosocial well-being of university students in Hong Kong. Public Health Nursing, 22(3), 209-220 doi:10.1111/ j.0737-1209.2005.220304.x

Lindfors, P., Berntsson, L., \& Lundberg, U. (2006). Factor structure of Ryff's psychological we1l-being scales in Swedish female and male white-collar workers. Personality and Individual Differences, 40, 1213-1222.

Londoño, A. N. H., Rogers, H. L., Castilla, T. J. F., Posada, G. S. L., Ochoa, A. N. L., Jaramillo, P. M. Á., \& Aguirre, A. D. C. (2012). Validacion en Colombia del cuestionario MOS de apoyo social. International Journal of Psychological Research, 5(1), 142-150.

Lin, N., Woelfel, M. W., \& Light, S. C. (1985). The buffering effect of social support subsequent to an important life event. Journal of Health \& Social Behavior, 26(3), 247-263.

Rodríguez-Carvajal, R., Díaz Méndez, D., Moreno-Jiménez, B., Blanco Abarca, A., \& van Dierendonck, D. (2010). Vitalidad y recursos internos como componentes del constructo to de bienestar psicológico. Psicothema, 22(1), 63-70.

Rodríguez-Espinola, S., \& Enrique, H. C. (s. d.). Validación Argentina del cuestionario MOS de apoyo social percibido. Psicodebate. Psicologia, Cultura y Sociedad, 7, 155-168. Recuperado de http://www.palermo.edu/cienciassociales/ publicaciones/pdf/Psico7/7Psico 10.pdf

Rosa-Rodríguez, Y., \& Quiñonez-Berrios, A. (2013). El bienestar psicológico en el proceso de ayuda con estudiantes universitarios. Revista Griot, 5(1), 7-17. Recuperado de http://ojs.uprrp.edu/ index.php/griot/article/viewArticle/279

Ryan, R. M., \& Deci, E. L. (2001). On happiness and human potentials: a review of research on hedonic and eudaimonic well-being. Annual Review of Psychology, 52(1), 141-166.

Ryff, C. (2013). Psychological well-being revisited: Advances in the science and practice of eudaimonia. Psychotherapy and Psychosomatics. Recuperado de http://www.karger.com/Article/ FullText/353263

Ryff, C. D. (1989). Happiness is everything, or is it? explorations on the meaning of psychological well-being. Journal of Personality \& Social Psychology, 57(6), 1069-1081.

Ryff, C., Keyes, C., \& Hughes, D. (2003). Status inequalities, perceived discrimination, and eudaimonic well-being: Do the challenges of minority life hone purpose and growth? Journal of Health and Social Behavior, 275-291

Sherbourne, C. D., Stewart, \& A. L. (1991). The MOS social support survey. Social Science \& Medicine, 32(6), 705-714. doi:10.1016/02779536(91)90150-b

Torres, F., Pompa, E., Meza, C., Ancer, L., \& González, M. (2010). Relación entre auto concepto y apoyo social en estudiantes universitarios. International Journal of Good Conscience, 5(2), 298-307.

Van Dierendonck, D., Díaz, D., Rodríguez-Carvajal, R., Blanco, A., \& Moreno-Jiménez, B. (2007). Ryff's six-factor model of psychological we- 
1l-being: A Spanish exploration. Social Indicators Research, 87(3), 473-479. doi:10.1007/ s11205-007-9174-7

Velásquez, C., Montgomery, W., Montero, V., Pomalaya, P., Dioses, A., Velasquez, N., \& Reynoso, D. (2008). Bienestar Psicológico, asertividad y rendimiento académico en estudiantes universitarios Sanmarquinos. Revista IIPSI, 11(2), $139-152$.
Vivaldi, F., \& Barra, E. (2012). Bienestar Psicológico, apoyo social percibido y percepción de salud en adultos mayores. Terapia Psicológica, 30(2), 23-29.

Wang, C. C. D. C., \& Castañeda-Sound, C. (2008). The role of generational status, self-esteem, academic self-efficacy and perceived social support in college students' psychological well-being. Journal of College Counseling, 11(2), 101-118.

Fecha de recepción: 13 de septiembre de 2013 Fecha de aceptación: 26 de junio de 2014 
